\title{
Quantitative stress analysis by means of standard infrared thermogaphic equipment
}

\author{
by S. Offermann1, C. Bissieux¹, J.L. Beaudoin1, H. Frick²
}

1 Université de REIMS Champagne-Ardenne, Laboratoire d'Energétique et d'Optique, Moulin de la Housse, B.P. 1039, F- 51687 REIMS CEDEX 2, e-mail: jl.beaudoin@univ-reims.fr; ${ }^{2}$ Hochschule für Technik und Wirtschaft des Saarlandes, Goebenstr. 40, D-66117 Saarbrücken

\begin{abstract}
In dynamic fatigue testing experiments, the thermoelastic effect links the local stress level and the local temperature variation in a material. Though quite minute, this effect can be readily measured by standard infrared thermography. Since neglecting the heat conduction is not relevant in the case of good conductors at high excitation frequencies, an adiabaticity criterion is established. An inverse technique to restore the thermally attenuated contrasts is proposed, using the finite element method for the direct heat conduction modelling. This technique has successfully be applied to an academical test sample. Measurements performed on some automotive parts under cyclic loading are presented. Besides, the influence of highly emissive coatings will be shortly discussed.
\end{abstract}

\section{Introduction}

In spite of large progresses in numerical modelisation of mechanical stresses in a loaded structure, experimental measurements are still needed. Thermoelastic stress analysis by means of radiometry consists in measuring with a scanning infrared radiometer the very small temperature variations of a sample under mechanical loading, then in calculating a stress map using an adequate model of the thermoelastic coupling. The theory of the thermoelastic effect, initiated by WEBER [1] and KELVIN [2] for isotropic homogeneous materials under adiabatic conditions has been extended by BIOT [3] and ROCCA and BEVER [4] within the framework of modern mechanical and thermodynamical theory. Within the elastic ranges, when a material is submitted to tensile or compressive stresses, it experiences reversible negative or positive temperature variations. These temperature variations are quite small $(\approx 1 \mathrm{mK}$ for $1 \mathrm{MPa}$ stress in mild steel). The first aim of this study has been the measurement of the thermoelastic stress field pattern by means of a wide-spread standard IR equipment like AGEMA 880 LW. The required noise rejection is obtained by a statistical procedure $[5,6]$ bringing the advantage that no synchronization link is needed between the test machine and the infrared equipment. It can be readily adapted to any infrared thermography apparatus. A thermal resolution of about $2 \mathrm{mK}$, corresponding to a stress resolution of about $2 \mathrm{MPa}$ for mild steel, is achieved in less than 5 minutes in industrial tests. Applications to some automotive parts, a motor crank rod and a steel wheel, are presented. Usually, in thermoelastic stress analysis the hypothesis of adiabaticity is applied, neglecting the heat conduction within the sample [7-10]. If quite true at high loading frequencies or with poorly conducting materials, it is much more approximative in most actual tests. For an academic sample the influence of heat diffusion has be quantified by means of a finite element model of the thermoelastic coupling concording to experimental results. We propose several inverse techniques to restore the thermally attenuated contrasts under nonadiabatic conditions and present the results obtained with one of them. Besides, the influence of highly emissive coatings will be shortly discussed.

\section{Theoretical considerations}

The widely used equation of the thermoelastic response [7]:

$$
\delta \mathrm{T}=-\frac{\alpha}{\rho c_{\mathrm{p}}} \mathrm{T}_{\mathrm{O}} \sigma_{\mathrm{I}}
$$

links local stresses and temperature variations by a linear equation. This simplification is only justified for high excitation frequencies or poor thermal conductors like polymers. In the case of good conductors like metals, the heat transfer attenuates the spatial temperature gradients. For a sample under harmonic loading, the relationship between stress and temperature variations is given by the solution of the nonhomogeneous differential equation: 


$$
\Delta T-\frac{j \omega}{a} T=-\frac{q}{k} \quad \text { or } \quad \Delta T-\frac{2 j}{\mu^{2}} T=-\frac{q}{k}
$$

where $q$ is the thermoelastic heat source and $\mu$ is the thermal diffusion length:

$$
\mathrm{q}=-\mathrm{j} \omega \alpha \mathrm{T}_{\mathrm{o}} \sigma_{\mathrm{I}} \quad \mu=\sqrt{\frac{2 \mathrm{a}}{\omega}}=\sqrt{\frac{\mathrm{a}}{\pi \mathrm{f}}}
$$

The adiabaticity hypothesis supposes the conduction fluxes to be neglected with respect to the heating sources:

$$
|\Delta \mathrm{T}| \ll\left|\frac{2 \mathrm{j}}{\mu^{2}} \mathrm{~T}\right| \quad \text { or } \quad|\Delta \mathrm{T}| \ll\left|\frac{\mathrm{q}}{\mathrm{k}}\right|
$$

So, we can derive an adiabaticity criterion related to the thermoelastic heat source i.e. to the stress:

$$
\mu^{2} \ll\left|2 j \frac{q}{\Delta q}\right|=2\left|\frac{q}{\Delta q}\right| \quad ; \quad \mu \ll \sqrt{2\left|\frac{\sigma_{I}}{\Delta \sigma_{1}}\right|}
$$

This criterion links the thermal diffusion length $\mu$ to the spatial variations of the principal stress sum $\sigma_{\mathrm{I}}$. In the case of good conductors and at low frequencies, this criterion is not satisfied, and the heat transfer attenuates the spatial temperature gradients. In order to get nevertheless reliable stress field patterns, inverse heat conduction calculations are required.

As mentioned above, when replacing in equation 1 the thermophysical parameters by their numerical values we note that the thermoelastic coupling is very low.

Another point is to be considered. As far as metals are poor infrared emitters, a black paint coating is necessary. It can be shown that the thermoelastic sources within the coating are negligible. But the coating behaves like a thermal barrier between the material under test and the infrared radiometer. This effect increases with the loading frequency and can lead to a strong underestimation of the stress amplitude. Theoretical calculations as well as experimental measurements have led us to the conclusion that coating thicknesses thinner than 20 micrometers are required in order to obtain good measurements at some tens of Hertz. Figure 1 shows the attenuation ratio between the temperature at the sample-coating interface and the surface temperature of the paint as a function of the coating thickness. These results were determined for a steel sample $\left(\mathrm{k}=50 \mathrm{~W} \cdot \mathrm{m}^{-1} \cdot \mathrm{K}^{-1} ; \rho=7860 \mathrm{~kg} \cdot \mathrm{m}^{3} ; \mathrm{C}=2000 \mathrm{~J} \cdot \mathrm{kg}^{-1} \cdot \mathrm{K}^{-1}\right)$ coated with a black paint $\left(k=0,25 W \cdot \mathrm{m}^{-1} \cdot \mathrm{K}^{-1} ; \rho=2000 \mathrm{~kg} \cdot \mathrm{m}^{3} ; \mathrm{C}=460 \mathrm{~J} \cdot \mathrm{kg}^{-1} \cdot \mathrm{K}^{-1}\right)$.

\section{Principle of operation - Experimental procedure}

The experimental set-up is shown on figure 2. As mentioned above, a thermal resolution of about $1 \mathrm{mK}$ is equivalent to a "stress resolution" of $\approx 1 \mathrm{MPa}$ in mild steel. This is far beyond the thermal resolution of common scanning infrared radiometers $\Leftrightarrow=0,1$ à $0,05 \mathrm{~K}$, at a rate of $\approx 30$ frames/s) . Drastic reduction of noise is thus essential.

The most commonly used technique is the well known "lock-in" detection which, by multiplying the noisy signal by a reference signal from the loading machine, strongly rejects uncorrelated noise. Successful applications of the lock-in technique have been reported, as well as for the dedicated SPATE from OMETRON [7] as with standard IR thermography equipments $[5,6,9,10]$.

Our own approach is different as it is based upon a statistical treatment of a series of thermograms recorded without synchronization to the loading force [5,6]. In a first step a series of infrared images of the unloaded sample is stored to a microcomputer. Then, we take another series of the same object under cyclic mechanical loading. The statistical analysis of these two series furnishes the temperature amplitude and thus the stress amplitude at each point of the loaded sample. Through an adequate choice of the statistical estimator, it is possible to derive the amplitude of the thermoelastic signal for various shapes of the time-dependence of the loading forces: purely harmonic, complex periodic, non periodic. The ability to treat various shapes of excitation sequences is certainly an advantage of this method. Another one is the extreme simplicity of the measurement procedure, as far as no synchronization is needed. One limitation of the method is that the phase of the thermoelastic signal is not obtained directly but should be derived from an additional fitting procedure.

The procedure has been first tested on a steel bar $\left(1 \times 1 \mathrm{~cm}^{2}\right.$ square section) with a $6 \mathrm{~mm}$ diameter hole drilled at its center. The well known "butterfly-like" stress pattern (figure 3) have 
been obtained. The thermal resolution was about $2 \mathrm{mK}$, equivalent to a stress resolution of about $2 \mathrm{MPa}$. A stress level of $25,3 \mathrm{MPa}$ is measured far from the hole, which is in good agreement with the experimental parameters (loading force $2,5 \mathrm{kN}, 1 \mathrm{~cm}^{2}$ cross-section of the bar).

These results have been obtained with a series of 750 IR frames, quantized on a 12 bit dynamic (PALMIR from ETNA-MECICA); the whole procedure (seizing + calculation), lasts only 5 min when implemented on a 486 DX/4-100 P.C..

\section{Finite element analysis}

In order to quantify the influence of heat diffusion in actual experimental conditions of thermoelastic stress analysis, we created a finite element model of the thermoelastic coupling by linking the mechanical and the thermal tools of a commercial software package (ANSYS 5.1 on HP $9000 / 715$ ). This thermal finite element calculation provides in the case of a simple academic sample the maps of temperature amplitudes (figures 4,5 ) as a function of the excitation frequency. Over the uniform stress region (at a distance from the hole), both the finite element analysis and the experiments gave a thermoelastic temperature amplitude of $25 \mathrm{mK}$, corresponding to a stress level of $25 \mathrm{MPa}$, according indeed to the ratio between the loading force and the sample section. This value does not depend on the excitation frequency, since the adiabatic hypothesis is always verified for uniform stress regions. On the other hand, for the high contrast regions (on both sides of the hole) the thermoelastic temperature amplitudes vary with the excitation frequency. Figure 6 shows the temperature amplitude along the hole section A-A for different excitation frequencies, but always with the same $2,5 \mathrm{kN}$ pure traction loading in the bar axis direction. At a frequency of $1000 \mathrm{~Hz}$, hence under adiabatic conditions, the profile of the temperature amplitudes is perfectly according to the real stress shape, but at $1 \mathrm{~Hz}$ the maximum temperature amplitude of $39 \mathrm{mK}$ is more than two times lower than 91 mK calculated at $1000 \mathrm{~Hz}$. Even at a frequency of $20 \mathrm{~Hz}$, commonly considered like high enough to neglect thermal diffusion, the spatial contrasts are still significantly attenuated in the stress concentration region.

\section{Inverse heat diffusion calculation}

Since the attenuation of high spatial gradients by thermal diffusion can be described by mathematical models, several inverse techniques can be proposed to correct the heat diffusion smoothing effect. Two groups of inversion methods can be distinguished. The first one consists in calculating the thermoelastic source distribution straight on from the bidimensional heat diffusion equation (2). This operation can also be achieved by deconvolution in the Fourier space.

Other techniques iterate the direct heat diffusion model until the calculated temperatures $T(x, y)$ converge close to the measured ones. Figure 7 shows the result of one of these techniques, where we used the finite element method for the direct model.

A first virtual source distribution $q_{1}$ is calculated, applying the adiabatic relationship to the measured temperature field $T_{0}$. Then, the direct model is applied to this source distribution, yielding a less contrasted temperature field $T_{1}$. Since the actual source distribution is more contrasted than $q_{1}$, a new source distribution $q_{2}$ is determined, more constrasted than $q_{1}$ :

$$
\mathrm{q}_{2}(\mathrm{x}, \mathrm{y})=\mathrm{q}_{1}(\mathrm{x}, \mathrm{y})+\rho \mathrm{C}_{\sigma} \omega\left(\left|\mathrm{T}_{0}(\mathrm{x}, \mathrm{y})\right|-\left|\mathrm{T}_{1}(\mathrm{x}, \mathrm{y})\right|\right)
$$

A new application of the direct model leads to a new distribution $T_{2}$, much closer to $T_{0}$ than $T_{1}$.

\section{Industrial applications}

Car steel wheel: Car wheels are commonly submitted to various fatigue testings. In the case that we report hereafter, the wheel was fixed on a massive support. A periodically rotating torque was applied to the wheel-center via a rotating shaft loaded with an excentric load at its other end. The excitation frequency was about $20 \mathrm{~Hz}$. The obtained stress pattern is shown on figure 8 . The amplitude of the torque was settled to $1,45 \mathrm{kN}$.m. It must be noted that the hole periphery patterns are not significant. They are due to the small vibration $(\sim 4 \mathrm{~mm})$ of the whole apparatus relatively to the IR camera. Customary procedures of object motion compensation could be used in order to rectify this imperfection.

Automotive motor crank bar (figure 9): The other tested part was a motor crank bar made from G.S. cast iron. It was submitted to harmonic uniaxial tensile loading (1 to $10 \mathrm{kN}$ ) by a SCHENK fatigue machine, at frequencies of 10 and $30 \mathrm{~Hz}$. Strain gauges disposed at the opposite side of the bar, approximately at the spot of highest stress concentration, indicated stress amplitudes higher by about $35 \%$ than that derived from thermoelastic measurements. Either the uncertainties in the values of the used physical parameters, or the three-dimensional heat transfer inside the sample, may be responsible for this discrepancy. 


\section{Conclusions}

Thermoelasticimetry does not pretend to substitute for finite element calculations or for strain gauge measurements. It should be considered as complementary for its unique property in a rapid, non-contact imaging of the whole field of (principal) stresses, which can be recorded without modification of the fatigue test procedure. Preparation reduces to spray black paint on the sample. The method that we have elaborated is quite simple, as it requires only a standard thermography equipment used with a software package. It has been tested successfully in real industrial tests. A stress resolution of about $2 \mathrm{MPa}$ is easily obtained in some minutes. However, only under adiabatic conditions the measured thermal amplitude map is proportional to the stress field pattern. In a number of real industrial tests this simplification cannot be asserted by the established adiabatic criterion. Under non-adiabatic conditions the thermal diffusion attenuates the spatial gradients on the thermoelastic stress maps. Consequently, some inverse techniques are welcome. The experimented inverse technique by iteration on the direct heat diffusion model yields a good restoration of the real stress map.

\section{REFERENCES}

[1] WEBER (W.). - Über die spezifische Wärme fester Körper insbesondere der Metalle. Annalen der Physik und Chemie, Vol. 96, 1830, p. $177-213$.

[2] THOMSON (W.) (Lord KELVIN). - On the dynamical theory of heat. Transactions of the Royal Society, Edinburgh, Vol. 20, 1853, p. 261-283.

[3] BIOT (M.A.). -Thermoelasticity and irreversible thermodynamics. Journal of Applied Physics, Vol. $27, N^{\circ} 3$, mars 1956, p. 240-253.

[4] ROCCA (R.) and BEVER (M.B.). - The Thermoelastic effect in iron and nickel as a function of temperature. Transactions AlME, Vol. 188, février 1950, p. 327 -333.

[5] OFFERMANN (S.), MERIENNE (E.), BEAUDOIN (J.L.). - Statistical method to evaluate the amplitude of cyclical noisy signal in IR Thermography. NATO ASI Series, Series E: Applied sciences - Vol. 262, 1993.

[6] OFFERMANN (S.), BISSIEUX (C.), BEAUDOIN (J.L.). - Thermoelastic stress analysis with standard Thermographic Equipment by means of statistical noise rejection. Research in Non destuctive Evaluation, Vol. 7, $\mathrm{N}^{\circ} 4,1996$, p. 239-251.

[7] MOUNTAIN (D.S.) and WEBBER (J.M.B.). - Stress Pattern Analysis by Thermal Emission (SPATE). SPIE Proc.167, 1978, p 189-196.

[8] STANLEY (P.) and CHAN (W.K.). - Quantitative Stress Analysis by Means of the Thermoelastic Effect. Journal of Strain Analysis, Vol. 20, $\mathrm{N}^{\circ} 3,1985, \mathrm{p} .129-137$.

[9] BLANC (R.) and GIACOMETTI (E.). - Infrared stroboscopy - a method for the study of thermomechanical behaviour of materials and structures at high rates of strain. Int. J. Solid Structures, Vol. 17, 1981, p. 531-540.

[10] BREMONT (P.). - La Thermographie Infrarouge pour voir les contraintes. Mesures, Vol. 673, mars 1995, p 53-56. [in French]

\section{FIGURES}

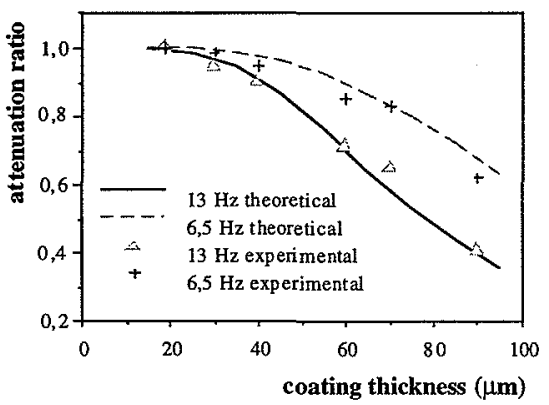

Figure 1: Influence of high emissivity coatings 


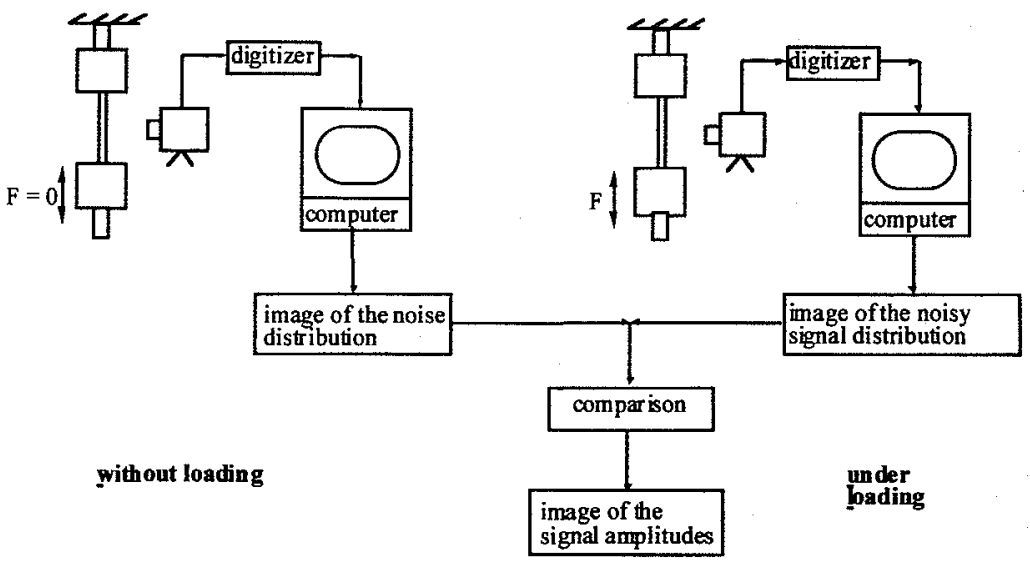

Figure 2: Scheme of the experimental setup

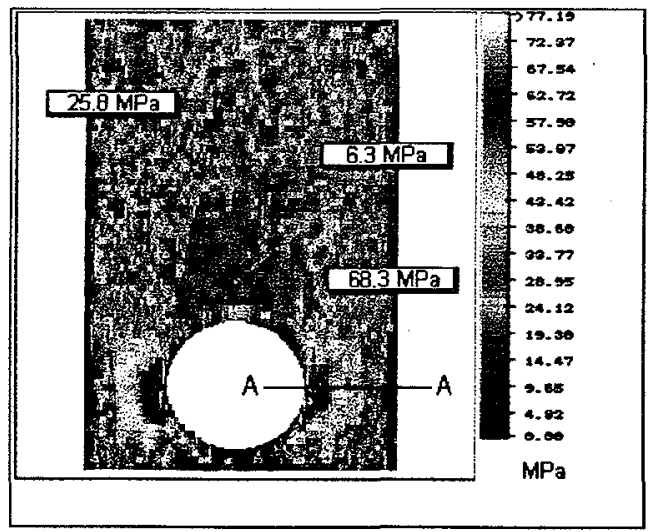

Figure 3: Experimental stress map at $20 \mathrm{~Hz}$
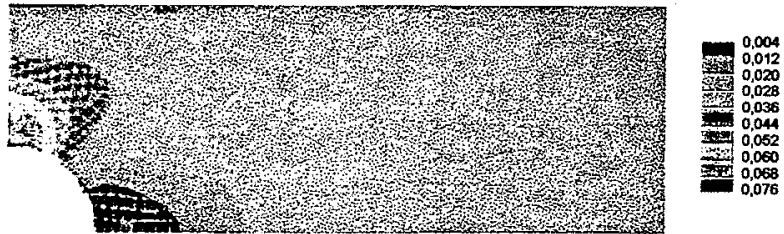

Figure 4: Calculated thermoelastic temperature $\operatorname{map}(\mathrm{K})$ at $20 \mathrm{~Hz}$

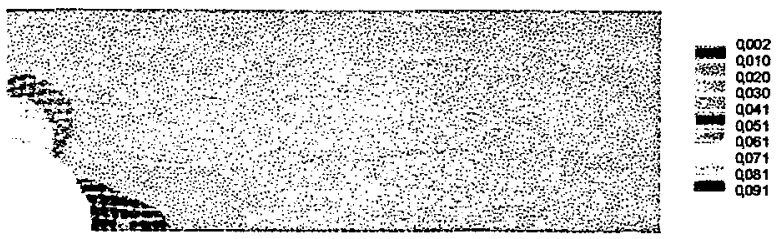

Figure 5: Calculated thermoelastic temperature map (K) at $1000 \mathrm{~Hz}$ 


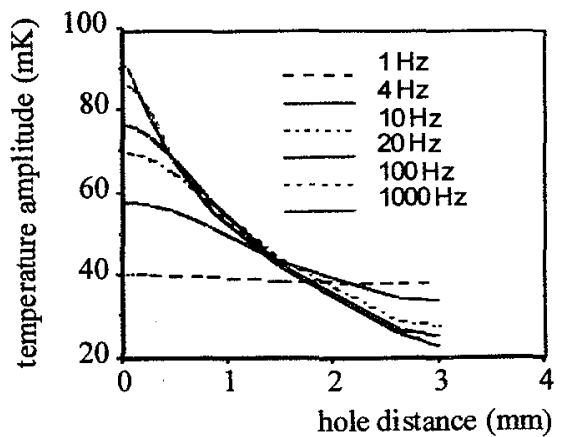

Figure 6:Temperature profiles in the hole section of the academical sample

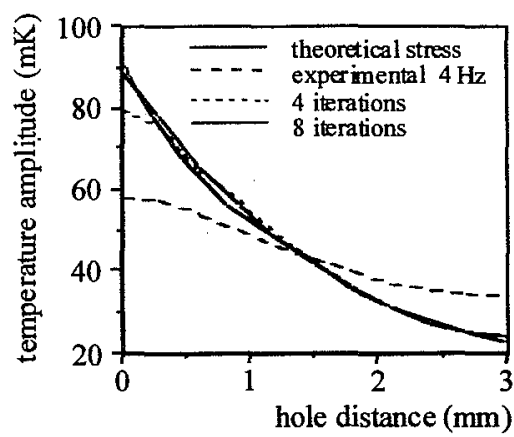

Figure 7: Correction of the attenuated stress profile

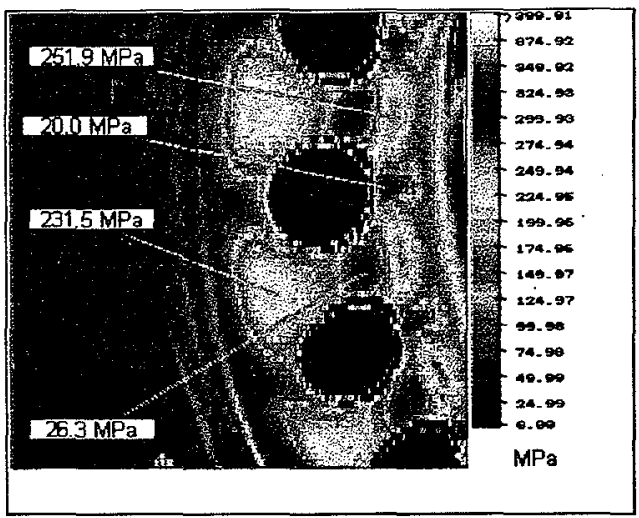

Figure 8: Thermoelastic stress map of a car wheel (Document L.E.O. - Dunlop)

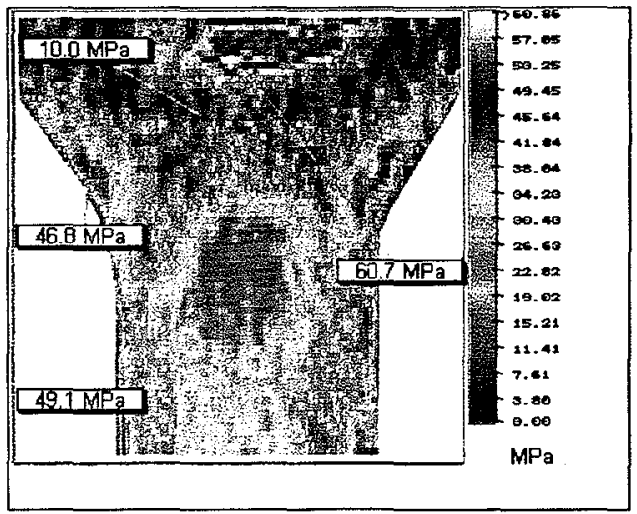

Figure 9: Thermoelastic stress map of motor crank bar (Document L.E.O. - P.S.A.) 